\title{
A SIMPLE MODEL FOR PHASE TRANSITIONS: FROM THE DISCRETE TO THE CONTINUUM PROBLEM
}

\author{
BY \\ S. PAGANO (LMGC, Université de Montpellier II-UMR 5508, Place Eugene Bataillon, 34005 \\ Montpellier Cedex, France) \\ AND
}

R. PARONI (Dipartimento di Ingegneria Civile, Universitá degli Studi di Udine, Via delle Scienze 208, 33100 Udine, Italy)

\begin{abstract}
In this paper we study a one-dimensional model simulating the shear in a two-dimensional body. We analyse the discrete system and we deduce the continuum limit of the lattice model as the lattice parameter goes to zero. Different energies are introduced and linked together.
\end{abstract}

1. Introduction. Recently there has been a strong and successful effort to use nonlinear elasticity for modelling phase transition, especially for the study of reversible phase transitions in shape memory alloys. Using a continuum theory, these works permit, in various cases, the explanation and description of the formation and variety of microstructures experimentally observed. In general, these approaches are based on the minimisation of a non-(quasi)convex elastic free energy. As explained by Ball and James [1, 2], the formation of microstructures reflects the fact that the energy is not lower semicontinuous with respect to weak convergence in $W^{1, p}$, which, in general, implies that there is no minimiser. The relaxed energy, instead, will be (quasi-) convex and will have minimisers.

Other works based on lattice models permit the study of phase transitions and the nonlinear dynamics of microstructure. These atomistic models involve nonlinear and competing interactions between the "atoms". Some lattice instabilities, which can be exhibited, can be used to explain the formation of microstructure and the existence of strain waves. In recent papers by Pouget [15, 16, 17] and Rosenau [21], some quasicontinuum models based on these atomistic descriptions are obtained. Some authors $[6,10$, $11,15,16,17]$ use the Fourier image of discrete functions to deduce, after expanding the interaction potential, a quasicontinuum model in which macroscopic and microscopic stresses can be derived from an appropriate elastic potential. In other works [21], the quasicontinuum model is obtained by inversion of an operator which is introduced by a

Received April 4, 2000.

2000 Mathematics Subject Classification. Primary 74Gxx, 74E15, 74N15, 49J45.

Key words and phrases. Phase transitions, discrete systems, double-well potentials, $\Gamma$-convergence. 
Taylor expansion. Other mathematical works use a very precise notion of convergence to pass from the discrete model to the continuum. To the best of our knowledge, Braides, Dal Maso, and Garroni [3] were the first to deduce a continuum theory starting from an atomistic description by means of $\Gamma$-convergence. In [3] the authors study the softening in fracture mechanics of a one-dimensional continua. Further studies in this direction were done by Braides and Gelli $[4,5]$ and in a stochastic setting by Iosifescu, Licht, and Michaille [8].

In this paper we consider a one-dimensional model simulating the shear in a twodimensional body described at a discrete level. We study the discrete system and we also deduce its continuum limit as the lattice parameter tends to zero.

In Sec. 2 the region occupied by the body is taken to be, for simplicity, the square $(0, l) \times(0, l)$. The reference configuration is made by a discrete system of $(n+1) \times(n+1)$ masses equally spaced with mutual distance $\lambda_{n}:=l / n$. In the lattice model, like in papers by Pouget $[10,15,16,17]$ and Novak and Salje $[12,13]$, each point interacts with its first, second, and third-nearest neighbours. We assume that all the atoms located in the same vertical layer have the same displacement and that this displacement is in a fixed direction; thus, the model reduces to a one-dimensional model. Under this assumption, which is made to simplify the problem, we show that the potential $W$ of the first nearest neighbour in the one-dimensional model is a combination of the first and the second potentials, $\varphi_{1}$ and $\varphi_{2}$, in the two-dimensional model. We further show, by taking $\varphi_{1}$ and $\varphi_{2}$ to be Lennard-Jones potentials, that $W$ is either a convex function or a double-well potential. In the rest of the sections a double-well potential is considered.

In Sec. 3 for the second-nearest neighbour we take the energy $\mathcal{W}$ convex and we study the discrete system. Following Puglisi and Truskinovsky [18], we define the equilibrium energies $\bar{E}_{n}$. Our analysis enables us to conclude that the form of the energy $\bar{E}_{n}$ depends strongly on $\mathcal{W}$ and has a lot of local minima, the number of which is related to the number of springs considered. We observe that as the number of springs becomes larger and larger the envelope of the energy $\bar{E}_{n}$ is getting closer and closer to an energy similar to the convexification of $\bar{E}_{2}$. This statement is made precise in Sec. 5 , specifically in Theorem 2, where we deduce the continuum limit of the model for a general function $\mathcal{W}$ (it could be neither convex nor concave). Such a continuum limit is derived by using $\Gamma$-convergence in a framework like the one used by Braides, Dal Maso, and Garroni [3]. The potential energy of the continuum model is convex; indeed the $\Gamma$-limit gives the convexification of the continuum energy. In this way we establish a link among the energies at different scales: the interatomic potential, the double-well potential which is a combination of interatomic potentials, the equilibrium energy, and the continuum one.

Finally in the conclusions, besides making some remarks, we briefly look at the case in which the energy $\mathcal{W}$ is concave. This case turns out to be very different from the case in which $\mathcal{W}$ is convex: in this latter case we have "oscillations" at the atomic level while in the former case, i.e., $\mathcal{W}$ concave, the number of interfaces is minimised.

2. Lattice Model. We consider a two-dimensional body which we identify with a region $\Omega$ of the two-dimensional Euclidean space. For simplicity, the region $\Omega$ is taken to be the square $(0, l) \times(0, l)$. The side $\{0\} \times(0, l)$ is clamped and the two sides $(0, l) \times\{0\}$ 


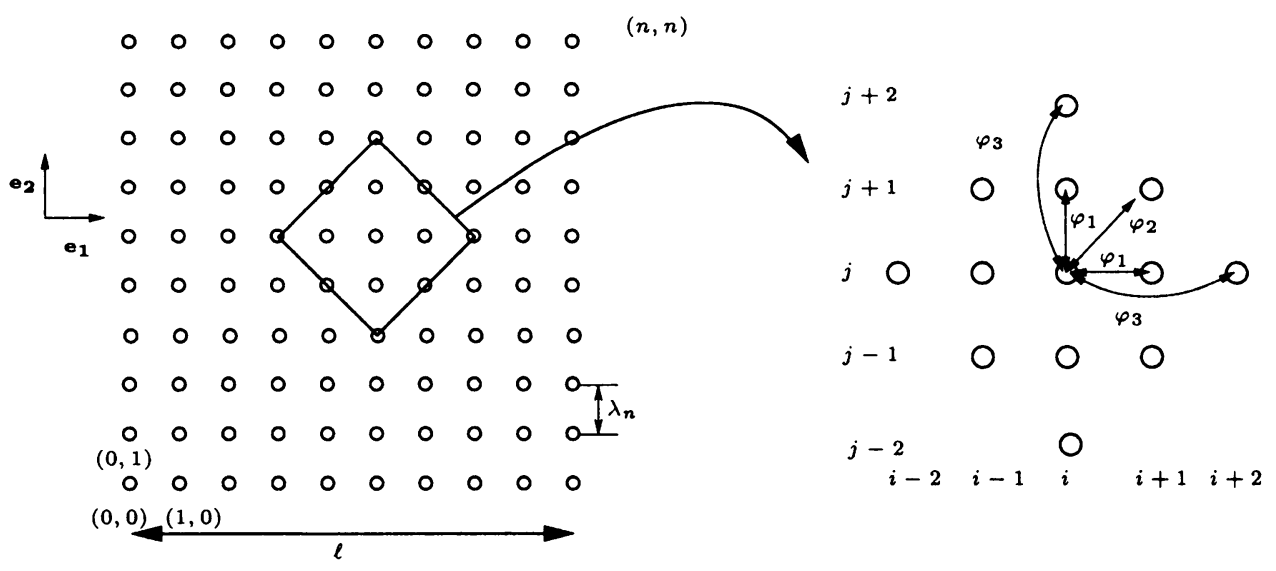

FIG. 1. Two-dimensional lattice energy. First-, second-, and thirdnearest neighbours

and $(0, l) \times\{l\}$ are free of boundary conditions. In the remaining side, a displacement $\mathbf{u}$ of type $\mathbf{u}=d \mathbf{e}_{2}$ is applied, where $d$ is constant and $\left(\mathbf{e}_{1}, \mathbf{e}_{2}\right)$ is a fixed orthonormal basis of $\mathbb{R}^{2}$. The material properties will be described later not at a continuum level but at an atomistic level by specifying the interatomic potentials between the "atoms". We suppose the reference configuration to be made by a discrete system of $(n+1) \times(n+1)$ masses located at the points (see Fig. 1) $\mathbf{X}_{n}(i, j)$ with

$$
\mathbf{X}_{n}(i, j)=\lambda_{n} i \mathbf{e}_{1}+\lambda_{n} j \mathbf{e}_{2}, \quad i, j=0, \ldots, n .
$$

The subscript $n$ indicates that we are dealing with $(n+1) \times(n+1)$ masses. In the two directions, all the points are equally spaced with mutual distance $\lambda_{n}:=l / n$. We denote by $\mathbf{x}_{n}(i, j)$ the placement after deformation of the particle $(i, j)$ and by $\mathbf{u}_{n}$ the displacement of this point, so that

$$
\mathbf{x}_{n}(i, j)=\mathbf{X}_{n}(i, j)+\mathbf{u}_{n}(i, j), \quad i, j=0, \ldots, n .
$$

At this discrete level, the boundary conditions become

$$
\mathbf{u}_{n}(0, j)=\mathbf{0}, \mathbf{u}_{n}(n, j)=d \mathbf{e}_{2}, \quad j=0, \ldots, n .
$$

We assume that each lattice point $(i, j)$ interacts with the nearest points located in the vertical and horizontal directions (first-nearest neighbours), with the nearest points in the two diagonals (second-nearest neighbours), and with the second-nearest neighbours in the vertical and horizontal directions (third-nearest neighbours) (see Fig. 1). In fact, we consider particle pairs interaction between the first, second, and third-nearest neighbours; these points "are connected by nonlinear springs" (see Fig. 1). Throughout the paper we use Novak and Salje's terminology for the first, second, and third-nearest neighbours; other authors $[6,10,11,15,16,17,21]$ call our first- and second-nearest neighbours simply first-nearest neighbours and our third-nearest neighbours are called second-nearest neighbours. We suppose that the two potentials, associated to the first and the second 
nonlinear springs, depend only on the modulus of the relative atom positions and we choose Lennard-Jones potentials in the two cases,

$$
\varphi_{i}(r)=\delta_{i}\left(\frac{c_{i}^{12}}{|r|^{12}}-2 \frac{c_{i}^{6}}{|r|^{6}}\right), \quad i=1,2
$$

with two different constants $c_{1}$ and $c_{2}$. The potential $\varphi_{3}$ for the third-nearest neighbours will be specified and discussed later.

With these notations, the total energy $E_{n}\left(\left\{\mathbf{u}_{n}\right\}\right)$ of the system is given by

$$
\begin{aligned}
E_{n}\left(\left\{\mathbf{u}_{n}\right\}\right):=\sum_{i=1}^{n} \sum_{j=0}^{n} \varphi_{1}\left(\left|\mathbf{x}_{n}(i, j)-\mathbf{x}_{n}(i-1, j)\right|\right)+\sum_{i=0}^{n} \sum_{j=1}^{n} \varphi_{1}\left(\left|\mathbf{x}_{n}(i, j)-\mathbf{x}_{n}(i, j-1)\right|\right) \\
\quad+\sum_{i=1}^{n} \sum_{j=1}^{n} \varphi_{2}\left(\left|\mathbf{x}_{n}(i, j)-\mathbf{x}_{n}(i-1, j-1)\right|\right)+\sum_{i=1}^{n} \sum_{j=0}^{n-1} \varphi_{2}\left(\left|\mathbf{x}_{n}(i, j)-\mathbf{x}_{n}(i-1, j+1)\right|\right) \\
\quad+\sum_{i=2}^{n} \sum_{j=0}^{n} \varphi_{3}\left(\left|\mathbf{x}_{n}(i, j)-\mathbf{x}_{n}(i-2, j)\right|\right)+\sum_{i=0}^{n} \sum_{j=2}^{n} \varphi_{3}\left(\left|\mathbf{x}_{n}(i, j)-\mathbf{x}_{n}(i, j-2)\right|\right)
\end{aligned}
$$

where the two first terms correspond to the first-nearest neighbour interactions in the horizontal and vertical directions, the third and fourth terms correspond to the secondnearest neighbour in the two diagonals, and the two last terms correspond to the thirdnearest neighbour interactions in the horizontal and vertical directions.

To simplify the problem we make the following assumption: not just the last vertical layer of masses will have a displacement like the one prescribed in Eq. (2) but all vertical layers. To be more precise, we shall study the case where $\mathbf{u}_{n}$ depends only on the index $i$ and only the component in the $\mathbf{e}_{2}$ direction is different from zero; i.e., all the particles in the vertical layer $i$ have the same displacement

$$
\mathbf{u}_{n}(i, j)=u_{n}^{i} \mathbf{e}_{2}, \quad i, j=0, \ldots, n
$$

Before continuing we would like to warn the reader that this is a mathematical assumption which may or may not be satisfied by a real system; a similar assumption was made by Pouget $[15,16]$. Actually, $u_{n}^{i}$ denotes the transversal displacement of the $i$ th layer of the lattice and in the following we will write for the displacement the only dependence on the indices of the vertical layer. 


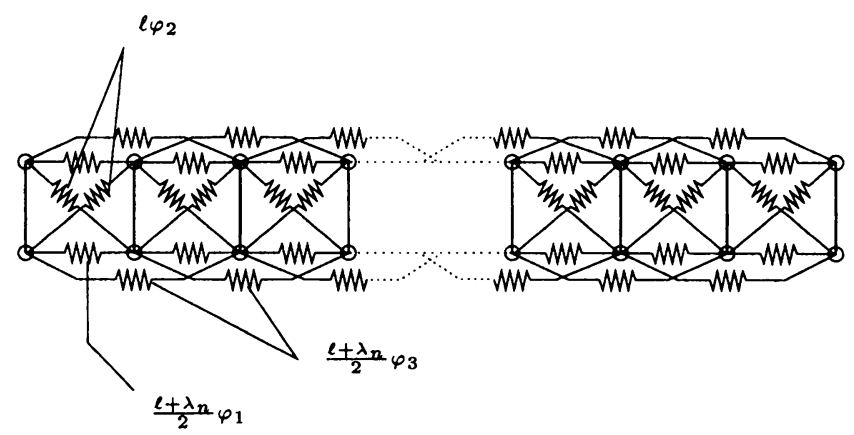

FIG. 2. Discrete pattern equivalent to the one represented in Fig. 1

Under this assumption the total energy can be rewritten as:

$$
\begin{aligned}
E_{n}\left(\left\{\varepsilon_{n}^{i}\right\}\right):= & \sum_{i=1}^{n} \sum_{j=0}^{n} \varphi_{1}\left(\lambda_{n} \sqrt{1+\varepsilon_{n}^{i^{2}}}\right)+\sum_{i=1}^{n} \sum_{j=1}^{n} \varphi_{2}\left(\lambda_{n} \sqrt{1+\left(1+\varepsilon_{n}^{i}\right)^{2}}\right) \\
& +\sum_{i=1}^{n} \sum_{j=0}^{n-1} \varphi_{2}\left(\lambda_{n} \sqrt{1+\left(1-\varepsilon_{n}^{i}\right)^{2}}\right)+\sum_{i=2}^{n} \sum_{j=0}^{n} \varphi_{3}\left(\lambda_{n} \sqrt{4+\left(\varepsilon_{n}^{i-1}+\varepsilon_{n}^{i}\right)^{2}}\right) \\
= & (n+1) \sum_{i=1}^{n} \varphi_{1}\left(\lambda_{n} \sqrt{1+\varepsilon_{n}^{i^{2}}}\right) \\
& +n \sum_{i=1}^{n}\left(\varphi_{2}\left(\lambda_{n} \sqrt{1+\left(1+\varepsilon_{n}^{i}\right)^{2}}\right)+\varphi_{2}\left(\lambda_{n} \sqrt{1+\left(1-\varepsilon_{n}^{i}\right)^{2}}\right)\right) \\
& +(n+1) \sum_{i=2}^{n} \varphi_{3}\left(\lambda_{n} \sqrt{4+\left(\varepsilon_{n}^{i-1}+\varepsilon_{n}^{i}\right)^{2}}\right)
\end{aligned}
$$

where $\varepsilon_{n}^{i}:=\left(u_{n}^{i}-u_{n}^{i-1}\right) / \lambda_{n}$ is the relative displacement between the $i$ th and the $(i-1)$ th vertical layers. By using the relation $l=\lambda_{n} n$, we can rewrite Eq. (5) as:

$$
\begin{aligned}
\lambda_{n} E_{n}\left(\left\{\varepsilon_{n}^{i}\right\}\right):= & 2 \sum_{i=1}^{n} \frac{l+\lambda_{n}}{2} \varphi_{1}\left(\lambda_{n} \sqrt{1+\varepsilon_{n}^{i^{2}}}\right) \\
& +\sum_{i=1}^{n}\left\{l \varphi_{2}\left(\lambda_{n} \sqrt{1+\left(1+\varepsilon_{n}^{i}\right)^{2}}\right)+l \varphi_{2}\left(\lambda_{n} \sqrt{1+\left(1-\varepsilon_{n}^{i}\right)^{2}}\right)\right\} \\
& +2 \sum_{i=2}^{n} \frac{l+\lambda_{n}}{2} \varphi_{3}\left(\lambda_{n} \sqrt{4+\left(\varepsilon_{n}^{i-1}+\varepsilon_{n}^{i}\right)^{2}}\right) .
\end{aligned}
$$

The expression on the right-hand side of the equation above can be considered to be the energy of the system displayed in Fig. 2 constituted of two horizontal layers of atoms; the vertical pairs of atoms are linked by a rigid bar and are constrained to move vertically. 


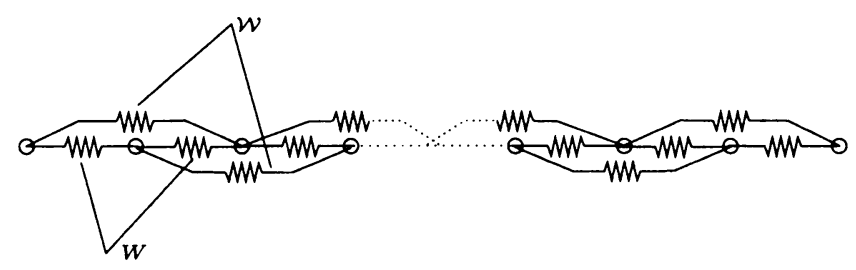

FIG. 3. One-dimensional layer of atoms equivalent to that in Fig. 2

We denote by $\psi_{i}$ the functions defined by

$$
\begin{aligned}
& \psi_{i}(r):=\varphi_{i}\left(\lambda_{n} \sqrt{r}\right)=\delta_{i}\left(\frac{k_{i}^{12}}{|r|^{6}}-2 \frac{k_{i}^{6}}{|r|^{3}}\right), \quad i=1,2, \\
& \psi_{3}(r):=\varphi_{3}\left(\lambda_{n} \sqrt{r}\right)
\end{aligned}
$$

where $k_{i}:=\frac{c_{i}}{\lambda_{n}}$. Here we have tacitly assumed that the ratio $k_{i}$ between the distance at equilibrium of two masses and the lattice spacing in the reference configuration does not depend on the number of masses considered. We can then rewrite Eq. (6) as follows:

$$
\begin{aligned}
\widetilde{E}_{n}\left(\left\{\varepsilon_{n}^{i}\right\}\right):= & \lambda_{n} E_{n}\left(\left\{\varepsilon_{n}^{i}\right\}\right) \\
= & \sum_{i=1}^{n} W\left(\varepsilon_{n}^{i}\right)+\sum_{i=2}^{n} \mathcal{W}\left(\varepsilon_{n}^{i-1}+\varepsilon_{n}^{i}\right) \\
& \quad+\frac{1}{n}\left(\sum_{i=1}^{n} G\left(\varepsilon_{n}^{i}\right)+\sum_{i=2}^{n} \mathcal{W}\left(\varepsilon_{n}^{i-1}+\varepsilon_{n}^{i}\right)\right),
\end{aligned}
$$

where

$$
\begin{aligned}
W(x) & :=l\left(\psi_{1}\left(1+x^{2}\right)+\psi_{2}\left(1+(1+x)^{2}\right)+\psi_{2}\left(1+(1-x)^{2}\right)\right), \\
\mathcal{W}(x) & :=l \psi_{3}\left(4+x^{2}\right), \\
G(x) & :=l \psi_{1}\left(1+x^{2}\right) .
\end{aligned}
$$

The sum of the first two terms on the right-hand side of Eq. (9) corresponds to the one-dimensional system presented in Fig. 3; in this case the longitudinal displacement of the atoms correspond to the vertical displacement in the two-dimensional model (see Fig. 2) and the energies for the first-nearest neighbour and the second-nearest neighbour, in the one-dimensional model, are respectively given by $W$ and $\mathcal{W}$.

The function $W$ is a combination of the potentials $\psi_{1}$ and $\psi_{2}$; to get an idea of what $W$ looks like, we will fix $\delta_{1}=\delta_{2}=1$ and $k_{1}=1$ as is done in the article by Novak and Salje $[12,13]$. The polynomial expansion of $W$ up to order four is

$$
W(x)=l\left(-\frac{3}{4} k_{2}^{6}+\frac{189}{128} k_{2}^{12}+9\right) x^{4}+l\left(-\frac{9}{4} k_{2}^{6}+\frac{9}{16} k_{2}^{12}\right) x^{2} .
$$

An elementary calculation shows that for $k_{2}$ greater than or equal to $\tilde{k}_{c}=\sqrt[3]{2}$, the function $W$ is convex, while for $k_{2}$ less than $\tilde{k}_{c}$, it is a double-well potential (see Fig. 4). The energy $W$ obtained looks like the Landau energy (see Fig. 4). Even with an expansion up to power higher than four we do not have an energy similar to the Devonshire energy. In the rest of the paper we will concentrate on the case of a double-well potential $W$. 


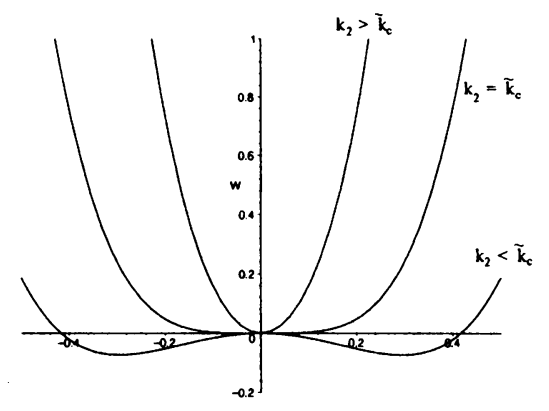

FIG. 4. Different $W$ for different $k_{2}$.

3. Analysis of the discrete system. In this section we study the discrete system, not only because it has some interest on its own, but also because we like to start to understand what the continuum energy should be. For this reason we restrict our attention, in this section, to the case of very simple functions $W$ and $\mathcal{W}$, so that we can actually compute the energies involved.

Throughout this section we mimic the analysis of Puglisi and Truskinovsky [18]; of course we will adapt their arguments to our case. For the reader's convenience we will restate what the main ideas are. We consider the energy $\widehat{E}_{n}$ defined by

$$
\widehat{E}_{n}\left(\left\{\varepsilon_{n}^{i}\right\}\right):=\sum_{i=1}^{n} W\left(\varepsilon_{n}^{i}\right)+\sum_{i=2}^{n} \mathcal{W}\left(\varepsilon_{n}^{i-1}+\varepsilon_{n}^{i}\right) .
$$

Essentially this energy is equal to the energy $\widehat{E}_{n}$ defined in Eq. (9), provided we neglect the term multiplied by $\frac{1}{n}$ (since $n$ goes to $+\infty$, for $n$ large enough these two energies will be approximately equal). In the next section we will go back to the general case. We study the following minimisation problem:

$$
\min \left\{\widehat{E}_{n}\left(\left\{\varepsilon_{n}^{i}\right\}\right): \sum_{i=1}^{n} \varepsilon_{n}^{i}=n \bar{\varepsilon}\right\},
$$

where, according to Eq. (2), $\bar{\varepsilon}$ is equal to $d / l$. To make the calculations simpler we take for $W$ a "tri-parabolic" expression, like in Puglisi and Truskinovsky [18], and for $\mathcal{W}$ a quadratic convex function,

$$
\begin{aligned}
& W(x):= \begin{cases}\frac{1}{2}(x+1)^{2} & \text { if } x<-2 / 5 \\
\frac{1}{2}\left(\frac{-3}{2} x^{2}+\frac{3}{5}\right) & \text { if }|x| \leq 2 / 5, \\
\frac{1}{2}(x-1)^{2} & \text { if } x>2 / 5\end{cases} \\
& \mathcal{W}(x):=\frac{1}{2} K x^{2},
\end{aligned}
$$

where $K$ is a positive constant; this, with the notation used in the previous section, corresponds to assume that $\psi_{3}$ is a linear function with positive slope. Again, in the next section we will go back to the general case. The energy $W$ is convex for $x$ less than $-2 / 5$ and for $x$ greater than $2 / 5$; we will refer to these two regions as phases I and III, respectively. These two convex wells are separated by a concave region which is 

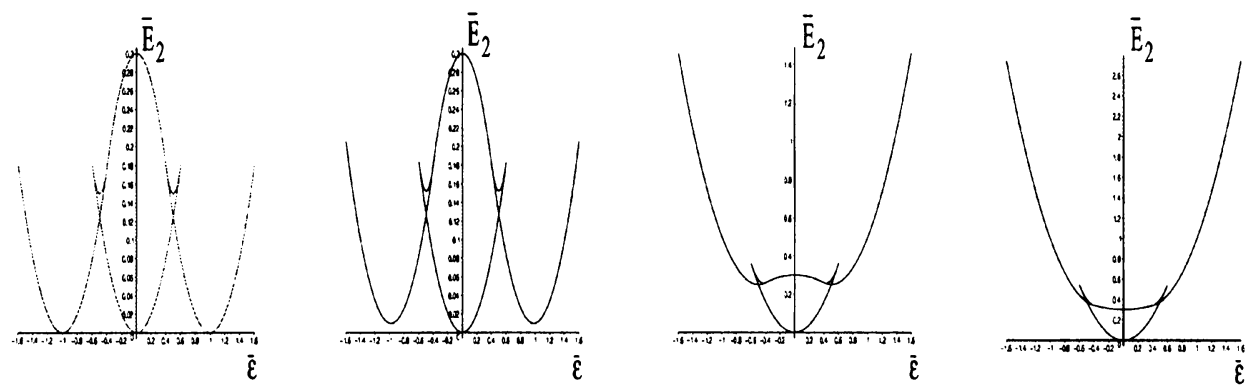

FIG. 5. Energies for a chain with two springs and with $K$ equal to $0,1 / 100,1 / 2$, and 1 , respectively

identified with phase II. By introducing the Lagrange multiplier $\bar{\sigma}$ (macroscopic stress), we can find the solution of our problem by minimising the unconstrained functional:

$$
\min \left(\widehat{E}_{n}\left(\left\{\varepsilon_{n}^{i}\right\}\right)-\bar{\sigma}\left(\sum_{i=1}^{n} \varepsilon_{n}^{i}-n \bar{\varepsilon}\right)\right) .
$$

The system of equilibrium equations

$$
\frac{\partial \widehat{E}_{n}}{\partial \varepsilon_{n}^{i}}=\bar{\sigma}, \quad i=1, \ldots, n,
$$

may have $3 \times n$ solutions, for every fixed $\bar{\varepsilon}$, since each of the springs can be in three different phases. Let $\varepsilon_{n}^{i}=f_{n}^{i}(\bar{\varepsilon})$ for $i=1, \ldots, n$, one of these $3 \times n$ solutions. We define a new "equilibrium" energy

$$
\bar{E}_{n}(\bar{\varepsilon})=\frac{1}{n} \widehat{E}_{n}\left(f_{n}^{1}(\bar{\varepsilon}), \ldots, f_{n}^{n}(\bar{\varepsilon})\right) .
$$

It can be easily checked that this equilibrium energy links the macro stress and the macro strain through the following equation:

$$
\frac{d \bar{E}_{n}}{d \bar{\varepsilon}}=\bar{\sigma}
$$

Of course, the energy $\bar{E}_{n}$ is not a function but is a multivalued function, since for every $\bar{\varepsilon}$ we may have, as said before, $3 \times n$ solutions; as a consequence, $\bar{\sigma}=\bar{\sigma}(\bar{\varepsilon})$ is also a multivalued function. Hereafter we report the energies that we have obtained for $n=2$ and $n=6$ (See Figures 5 and 7 ). The case $n=2$ is the simplest and, as we will see in the next section, in some sense, the most important.

In Fig. 5, different results obtained in the case of two springs, i.e., $n=2$, are shown. The case with $K=0$ is exactly the case studied by Puglisi and Truskinovsky [18]; in this case, the energy $\widehat{E}_{2}$ has four wells all at the same energy level. These four wells have the minima located at $\left(\varepsilon_{2}^{1}, \varepsilon_{2}^{2}\right) \in\{(-1,-1),(1,1),(-1,1),(1,-1)\}$, which correspond to the configurations represented in Fig. 6.

With the introduction of the third-nearest neighbours, i.e., when $K>0$, the energy level of the wells corresponding to the first two configurations in Fig. 6 is higher than that corresponding to the last two. Hence the system likes to alternate phase I and phase III, 

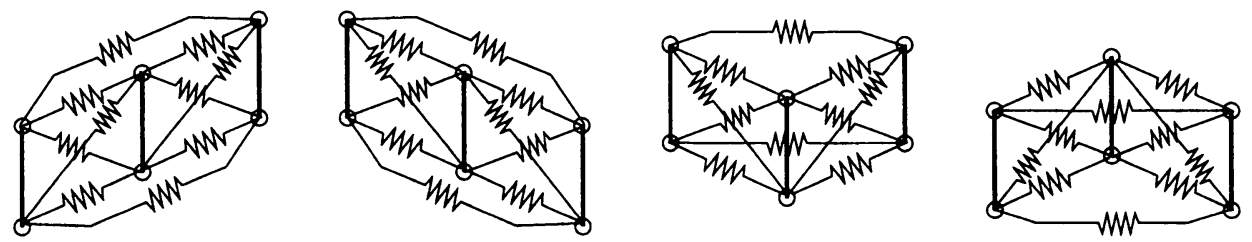

FiG. 6. Different local minimisers
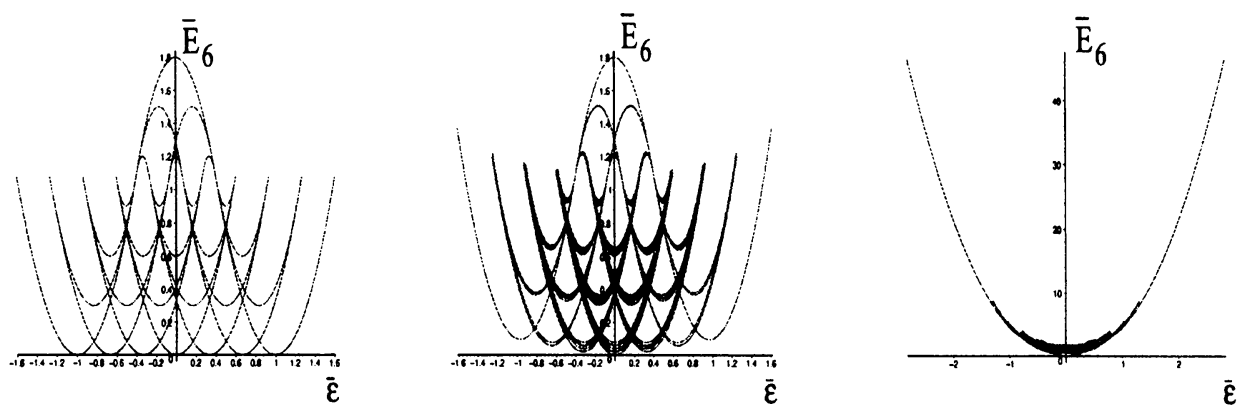

FIG. 7. Energies for a chain with six springs and with $K$ equal to 0 , $1 / 100$, and $1 / 2$, respectively

and thus we can already speculate that microstructure will be preferred, when possible, to a single phase. Roughly speaking, we can say that the third-nearest neighbours make a selection of the local minimisers. The envelope of $\bar{E}_{2}$ when $K=0$ (see Fig. 5) has three wells; in the first and the last one, i.e., those centered at $\bar{\varepsilon} \in\{-1,1\}$, respectively, both springs are in the same phase (I and III, respectively), while in the middle well a mixture of phases I and III appears. Phase II does not interfere with the envelope of $\bar{E}_{2}$.

The equilibrium energy when $K>0$ can be simply obtained (in the case $n=2$ ) by summing the equilibrium energy with $K=0$ with $1 / 2 \mathcal{W}(2 \bar{\varepsilon})$; hence, as $K$ becomes larger, the two wells centered at $\bar{\varepsilon}= \pm 1$ in the graph of the envelope of $\bar{E}_{2}$ when $K=0$ move upwards. As we can see from Fig. 5, the two wells disappear for $K$ large enough.

In Fig. 7 the equilibrium energies for a "chain" with six springs are shown. When $K=0$ we find again the same result of Puglisi and Truskinovsky [18]. Let us now comment on the case $K=1 / 100$. In this case, the graph of the envelope of $\bar{E}_{6}$ has seven wells or wiggles (loosely speaking we can say that it is a wiggly energy, cf. James [9]). The number of wiggles increases as we increase the number of masses $n$ (Truskinovsky and Zanzotto [22] found, in their model, something very similar). The well centered at $\bar{\varepsilon}=0$ is obtained when the springs alternate phase I and phase III. To indicate the "state" of the six springs we can use the following notation: $\left(i_{1}, i_{2}, \ldots, i_{6}\right)$ where $i_{j} \in\{1,2,3\}$ denotes the phase in which the $j$ th spring is. With this notation, the well centered at $\bar{\varepsilon}=0$ is in the state $(1,3,1,3,1,3)$ or equivalently $(3,1,3,1,3,1)$. As $\bar{\varepsilon}$ increases, the state will not change until we reach a point of "denucleation" after which there is a new well which is obtained when the springs are in $(3,1,3,1,3,3)$, for instance (another possibility is $(3,3,1,3,1,3))$. The next wiggle on the right instead is achieved at the 
state $(3,3,1,3,3,3)$, while the last well on the right is obtained when all the springs are in phase III, i.e., $(3,3,3,3,3,3)$. We see that as $\bar{\varepsilon}$ increases phase I tends to disappear ("denucleation"). A similar discussion can be done for the wells on the left of $\bar{\varepsilon}=0$. When $K=1 / 2$ the envelope of the equilibrium energy (see Fig. 7) looks like a convex function; this is because the effect of the third neighbour is "dominating" the effect of the first two. Indeed, also in the case $K=1 / 2$ the envelope of the equilibrium energy $\bar{E}_{6}$ is not convex; a magnification near the origin would show few wiggles.

Finally we observe that as $n$ becomes larger, for a fixed $K$, the envelope of $\bar{E}_{n}$ becomes closer and closer to the envelope of $\bar{E}_{2}$ (indeed to a rescaling of the envelope of $\bar{E}_{2}$, cf. Theorem 2).

4. Preliminaries- $-\Gamma$-convergence. Let $(X, d)$ be a metric space and $\left\{F_{h}\right\}_{h}$ and $F$ be functionals mapping $X$ into $\overline{\mathbb{R}}=\mathbb{R} \cup\{+\infty\}$. The following notion of convergence was first introduced by De Giorgi; for more details, we refer to the book of Dal Maso [7].

Definition 1. The sequence $\left\{F_{h}\right\}_{h} \Gamma$-converges to $F$ at $x$ in $X$ if and only if both of the following conditions are satisfied:

i) (liminf inequality) For every sequence $\left\{x_{h}\right\}_{h}$ of $X$, converging to $x$ in $X$, we have:

$$
F(x) \leq \liminf _{h \rightarrow+\infty} F_{h}\left(x_{h}\right) .
$$

ii) (recovery sequence) For every $x$ in $X$, there exists a sequence $\left\{x_{h}\right\}_{h}$ in $X$ such that

$$
F(x)=\lim _{h \rightarrow+\infty} F_{h}\left(x_{h}\right) .
$$

The main interest of this notion is explained in the following theorem.

Theorem 1. Assume that $\left\{F_{h}\right\}_{h} \Gamma$-converges to $F$ and $\left\{x_{h}\right\} \in X$ such that $F_{h}\left(x_{h}\right)=$ $\inf _{x \in X} F_{h}(x)$. If $x_{h}$ converges to $\bar{x}$, then we have

$$
F(\bar{x})=\inf _{x \in X} F(x)
$$

and

$$
F(\bar{x})=\lim _{h \rightarrow+\infty} F_{h}\left(x_{h}\right)
$$

5. Limit of the model. In this section we study the $\Gamma$-limit of the discrete model as the number of masses $n$ tends to infinity. The $\Gamma$-limit defines an energy, which we shall call continuum energy (indeed it should be called the convexification of the continuum energy), which can be used to identify the energetic value of the global minimisers; hence, all the information of local minimisers and barriers that were obtained in the previous section by studying the discrete system are not detained by the continuum energy.

We recall that the energy associated to the lattice model is

$$
\widetilde{E}_{n}\left(\left\{\varepsilon_{n}^{i}\right\}\right):=\sum_{i=1}^{n} W\left(\varepsilon_{n}^{i}\right)+\sum_{i=2}^{n} \mathcal{W}\left(\varepsilon_{n}^{i-1}+\varepsilon_{n}^{i}\right)+\frac{1}{n}\left(\sum_{i=1}^{n} G\left(\varepsilon_{n}^{i}\right)+\sum_{i=2}^{n} \mathcal{W}\left(\varepsilon_{n}^{i-1}+\varepsilon_{n}^{i}\right)\right)
$$

(see Eq. (9)), where $\varepsilon_{n}^{i}:=\left(u_{n}^{i}-u_{n}^{i-1}\right) / \lambda_{n}$ with $l=\lambda_{n} n$. In order to study the $\Gamma$-limit of the energy, we consider $\widetilde{E}_{n}$ as defined on functions and not simply on an array of numerical values. For this purpose, following Braides, Dal Maso, and Garroni [3], we 
introduce, for every $n$, the space $\mathcal{A}_{n}$ of continuous functions on $(0, l)$ which are affine on each interval $\left[X_{n}^{i}, X_{n}^{i+1}\right]=\left[i \lambda_{n},(i+1) \lambda_{n}\right]$ of $(0, l)$ for all $i$. In this way there is a oneto-one correspondence between $\mathcal{A}_{n}$ and the set of arrays $\left\{u_{n}^{i}\right\}_{i=1}^{n}: \mathbf{u}_{n} \in \mathcal{A}_{n}$ defines the array $\left\{u_{n}^{i}=\mathbf{u}_{n}\left(X_{n}^{i}\right)\right\}$, while if $\left\{u_{n}^{i}\right\}$ is an array, there exists only one function $\mathbf{u}_{n} \in \mathcal{A}_{n}$ such that $\mathbf{u}_{n}\left(X_{n}^{i}\right)=u_{n}^{i}$. Let $\mathbf{u}_{n} \in \mathcal{A}_{n}$ be the function associated to the array $\left\{u_{n}^{i}\right\}$; then, if we denote by $\dot{\mathbf{u}}_{n}$ the derivative of $\mathbf{u}_{n}$, we have that $\dot{\mathbf{u}}_{\left.n\right|_{\left(X_{n}^{i}, X_{n}^{i+1}\right)}}=\varepsilon_{n}^{i}$. We make the following assumptions of the energies, $W, \mathcal{W}$, and $G$ :

(H1) $\exists$ positive constants $c_{1}, c_{2}, C_{1}, C_{2}$ such that $c_{1}|\varepsilon|^{p}-c_{2} \leq W(\varepsilon) \leq C_{1}|\varepsilon|^{p}+C_{2}$.

(H2) $\exists$ positive constants $c_{3}, c_{4}, C_{3}, C_{4}$ such that $-c_{3}|\varepsilon|^{p}-c_{4} \leq \mathcal{W}(\varepsilon) \leq C_{3}|\varepsilon|^{p}+C_{4}$.

(H3) the constants above satisfy the inequality $c_{1}>2^{p} c_{3}$.

(H4) $\exists$ positive constants $c_{5}, c_{6}, C_{5}, C_{6}$ such that $-c_{5}|\varepsilon|^{p}-c_{6} \leq G(\varepsilon) \leq C_{5}|\varepsilon|^{p}+C_{6}$.

We consider the energy $\tilde{\mathcal{E}}_{n}$ defined on $L^{p}(0, l)$ by

$$
\widetilde{\mathcal{E}}_{n}\left(\mathbf{u}_{n}\right):= \begin{cases}\int_{0}^{l} W\left(\dot{\mathbf{u}}_{n}(x)\right) d x+\int_{\lambda_{n}}^{l} \mathcal{W}\left(\dot{\mathbf{u}}_{n}\left(x-\lambda_{n}\right)+\dot{\mathbf{u}}_{n}(x)\right) d x & \\ +\frac{1}{n}\left(\int_{0}^{l} G\left(\dot{\mathbf{u}}_{n}(x)\right) d x+\int_{\lambda_{n}}^{l} \mathcal{W}\left(\dot{\mathbf{u}}_{n}\left(x-\lambda_{n}\right)+\dot{\mathbf{u}}_{n}(x)\right) d x\right) & \text { if } \mathbf{u}_{n} \in \mathcal{A}_{n} \\ +\infty & \text { otherwise }\end{cases}
$$

Notice that if $\mathbf{u}_{n} \in \mathcal{A}_{n}$, then $\widetilde{\mathcal{E}}_{n}\left(\mathbf{u}_{n}\right)=\lambda_{n} \widetilde{E}_{n}\left(\left\{\varepsilon_{n}^{i}\right\}\right)$. Then we can prove

TheOrem 2. Assume (H1), (H2), (H3), and (H4). Then the energy functional $\widetilde{\mathcal{E}}_{n}$, defined in Eq. (17), $\Gamma$-converges to the functional $\widetilde{\mathcal{E}}$ defined in $L^{p}(0, l)$ by

$$
\widetilde{\mathcal{E}}(\mathbf{u})= \begin{cases}\int_{0}^{l} J^{* *}(\dot{\mathbf{u}}(x)) d x & \text { if } \mathbf{u} \in W^{1, p}(0, l) \\ +\infty & \text { otherwise }\end{cases}
$$

where $J(X):=\widetilde{W}(X)+\mathcal{W}(2 X), \widetilde{W}(X)=\frac{1}{2} \min \left\{W\left(X_{1}\right)+W\left(X_{2}\right): X_{1}+X_{2}=2 X\right\}$, and $J^{* *}$ is the convexification of $J$.

Proof. The proof is divided into two steps.

FIRST STEP: Proof of the "liminf inequality". Let a sequence $\left\{\mathbf{u}_{n}\right\}_{n}$ converge to $\mathbf{u}$ in $L^{p}(0, l)$. We want to prove that $\liminf \widetilde{\mathcal{E}}_{n}\left(\mathbf{u}_{n}\right) \geq \widetilde{\mathcal{E}}(\mathbf{u})$. If $\liminf \widetilde{\mathcal{E}}_{n}\left(\mathbf{u}_{n}\right)=+\infty$, then there is nothing to prove. Hence, without loss in generality, we suppose

$$
\liminf \widetilde{\mathcal{E}}_{n}\left(\mathbf{u}_{n}\right)<+\infty
$$


and $\mathbf{u}_{n} \in \mathcal{A}_{n}$. By using the definition of $\widetilde{\mathcal{E}}_{n}$ and the assumptions we find:

$$
\begin{aligned}
\widetilde{\mathcal{E}}_{n}\left(\mathbf{u}_{n}\right)= & \int_{0}^{l} W\left(\dot{\mathbf{u}}_{n}(x)\right) d x+\int_{\lambda_{n}}^{l} \mathcal{W}\left(\dot{\mathbf{u}}_{n}\left(x-\lambda_{n}\right)+\dot{\mathbf{u}}_{n}(x)\right) d x \\
& +\frac{1}{n}\left(\int_{0}^{l} G\left(\dot{\mathbf{u}}_{n}(x)\right) d x+\int_{\lambda_{n}}^{l} \mathcal{W}\left(\dot{\mathbf{u}}_{n}\left(x-\lambda_{n}\right)+\dot{\mathbf{u}}_{n}(x)\right) d x\right) \\
\geq & c_{1} \int_{0}^{l}\left|\dot{\mathbf{u}}_{n}(x)\right|^{p} d x-c_{2} l-c_{3} \int_{\lambda_{n}}^{l}\left|\dot{\mathbf{u}}_{n}\left(x-\lambda_{n}\right)+\dot{\mathbf{u}}_{n}(x)\right|^{p} d x-c_{4} l \\
& \quad-\frac{1}{n}\left(c_{5} \int_{0}^{l}\left|\dot{\mathbf{u}}_{n}(x)\right|^{p} d x+c_{6} l+c_{3} \int_{\lambda_{n}}^{l}\left|\dot{\mathbf{u}}_{n}\left(x-\lambda_{n}\right)+\dot{\mathbf{u}}_{n}(x)\right|^{p} d x+c_{4} l\right) \\
\geq & c_{1}\left\|\dot{\mathbf{u}}_{n}\right\|_{L^{p}}^{p}-2^{p-1} c_{3}\left(\int_{\lambda_{n}}^{l}\left|\dot{\mathbf{u}}_{n}\left(x-\lambda_{n}\right)\right|^{p} d x+\int_{\lambda_{n}}^{l}\left|\dot{\mathbf{u}}_{n}(x)\right|^{p} d x\right) \\
& \quad-\frac{1}{n}\left(c_{5}\left\|\dot{\mathbf{u}}_{n}\right\|_{L^{p}}^{p}+2^{p-1} c_{3}\left(\int_{\lambda_{n}}^{l}\left|\dot{\mathbf{u}}_{n}\left(x-\lambda_{n}\right)\right|^{p} d x+\int_{\lambda_{n}}^{l}\left|\dot{\mathbf{u}}_{n}(x)\right|^{p} d x\right)\right) \\
& \quad-l\left(c_{2}+c_{4}+\frac{c_{4}+c_{6}}{n}\right) \\
\geq & \left(c_{1}-2^{p} c_{3}-\frac{c_{5}+2^{p} c_{3}}{n}\right)\left\|\dot{\mathbf{u}}_{n}\right\|_{L^{p}}^{p}-l\left(c_{2}+c_{4}+\frac{c_{4}+c_{6}}{n}\right)
\end{aligned}
$$

so, for $n$ large enough,

$$
\widetilde{\mathcal{E}}_{n}\left(\mathbf{u}_{n}\right) \geq \frac{c_{1}-2^{p} c_{3}}{2}\left\|\dot{\mathbf{u}}_{n}\right\|_{L^{p}}^{p}-2 l\left(c_{2}+c_{4}\right)
$$

By hypothesis, $c_{1}-2^{p} c_{3}>0$; hence, from Eqs. (19) and (20), we have that, up to a subsequence, $\sup \left\|\dot{\mathbf{u}}_{n}\right\|_{L^{p}}<+\infty$, the sequence $\left\{\mathbf{u}_{n}\right\}_{n}$ converges weakly to $\mathbf{u}$ in $W^{1, p}(0, l)$, and, of course, $\mathbf{u}$ belongs to $W^{1, p}(0, l)$. We easily find that

$$
\begin{aligned}
& \frac{1}{n}\left|\int_{0}^{l} G\left(\dot{\mathbf{u}}_{n}(x)\right) d x+\int_{\lambda_{n}}^{l} \mathcal{W}\left(\dot{\mathbf{u}}_{n}\left(x-\lambda_{n}\right)+\dot{\mathbf{u}}_{n}(x)\right) d x\right| \\
& \leq \frac{1}{n}\left(\left(C_{1}+2^{p} C_{3}\right)\left\|\dot{\mathbf{u}}_{n}\right\|_{L^{p}}^{p}+\left(C_{4}+C_{6}\right) l\right),
\end{aligned}
$$

hence

$$
\frac{1}{n}\left(\int_{0}^{l} G\left(\dot{\mathbf{u}}_{n}(x)\right) d x+\int_{\lambda_{n}}^{l} \mathcal{W}\left(\dot{\mathbf{u}}_{n}\left(x-\lambda_{n}\right)+\dot{\mathbf{u}}_{n}(x)\right) d x\right)=o(1)
$$

i.e., the limit as $n$ goes to $+\infty$ of the left-hand side of the equation above is equal to zero.

We now find a lower bound for the energy $\widetilde{\mathcal{E}}_{n}$. We learned the technique that we are going to use in a conference by A. Braides on "Asymptotic Analysis for Non-convex Discrete Systems" held at Oxford in December 1999. 
The energy $\widetilde{\mathcal{E}}_{n}$ can be rewritten as

$$
\begin{aligned}
\widetilde{\mathcal{E}}_{n}\left(\mathbf{u}_{n}\right)= & \sum_{i=1}^{n} \lambda_{n} W\left(\varepsilon_{n}^{i}\right)+\sum_{i=2}^{n} \lambda_{n} \mathcal{W}\left(\varepsilon_{n}^{i-1}+\varepsilon_{n}^{i}\right)+o(1) \\
= & \sum_{\substack{i=2 \\
i \text { even }}}^{n} \frac{\lambda_{n}}{2} W\left(\varepsilon_{n}^{i-1}\right)+\frac{\lambda_{n}}{2} W\left(\varepsilon_{n}^{i}\right)+\sum_{\substack{i=2 \\
i \text { even }}}^{n} \lambda_{n} \mathcal{W}\left(\varepsilon_{n}^{i-1}+\varepsilon_{n}^{i}\right) \\
& +\sum_{\substack{i=3 \\
i \text { odd }}}^{n} \frac{\lambda_{n}}{2} W\left(\varepsilon_{n}^{i-1}\right)+\frac{\lambda_{n}}{2} W\left(\varepsilon_{n}^{i}\right)+\sum_{\substack{i=3 \\
i \text { odd }}}^{n} \lambda_{n} \mathcal{W}\left(\varepsilon_{n}^{i-1}+\varepsilon_{n}^{i}\right) \\
& +\frac{\lambda_{n}}{2} W\left(\varepsilon_{n}^{1}\right)+\frac{\lambda_{n}}{2} W\left(\varepsilon_{n}^{n}\right)+o(1) .
\end{aligned}
$$

By assumption (H1) we have $\frac{\lambda_{n}}{2} W\left(\varepsilon_{n}^{1}\right)+\frac{\lambda_{n}}{2} W\left(\varepsilon_{n}^{n}\right) \geq-c_{2} \lambda_{n}$, so we obtain

$$
\begin{aligned}
\widetilde{\mathcal{E}}_{n}\left(\mathbf{u}_{n}\right) \geq & \sum_{\substack{i=2 \\
i \text { even }}}^{n} \lambda_{n}\left\{\widetilde{W}\left(\frac{\varepsilon_{n}^{i-1}+\varepsilon_{n}^{i}}{2}\right)+\mathcal{W}\left(\varepsilon_{n}^{i-1}+\varepsilon_{n}^{i}\right)\right\} \\
& +\sum_{\substack{i=3 \\
i \text { odd }}}^{n} \lambda_{n}\left\{\widetilde{W}\left(\frac{\varepsilon_{n}^{i-1}+\varepsilon_{n}^{i}}{2}\right)+\mathcal{W}\left(\varepsilon_{n}^{i-1}+\varepsilon_{n}^{i}\right)\right\}+o(1) \\
= & \sum_{\substack{i=2 \\
i \text { even }}}^{n} \lambda_{n} J\left(\frac{\varepsilon_{n}^{i-1}+\varepsilon_{n}^{i}}{2}\right)+\sum_{i=3}^{n} \lambda_{n} J\left(\frac{\varepsilon_{n}^{i-1}+\varepsilon_{n}^{i}}{2}\right)+o(1) \\
= & \sum_{i=2}^{n} \lambda_{n} J\left(\frac{\varepsilon_{n}^{i-1}+\varepsilon_{n}^{i}}{2}\right)+o(1) \\
= & \int_{\lambda_{n}}^{l} J\left(\frac{\dot{\mathbf{u}}_{n}\left(x-\lambda_{n}\right)+\dot{\mathbf{u}}_{n}(x)}{2}\right) d x+o(1) .
\end{aligned}
$$

Hence, by definition of the convexification we have the following inequality:

$$
\widetilde{\mathcal{E}}_{n}\left(\mathbf{u}_{n}\right) \geq \int_{\lambda_{n}}^{l} J^{* *}\left(\frac{\dot{\mathbf{u}}_{n}\left(x-\lambda_{n}\right)+\dot{\mathbf{u}}_{n}(x)}{2}\right) d x+o(1) .
$$

We now choose a partition $p_{k}:=\left\{y_{0}, y_{1}, \ldots, y_{k}\right\}$ of $(0, l)$ and we define an integer $r(n):=\min \left\{j: y_{j} \geq \lambda_{n}\right\}+1$, so that

$$
\begin{aligned}
\widetilde{\mathcal{E}}_{n}\left(\mathbf{u}_{n}\right) & \geq \sum_{j=r(n)}^{k} \int_{y_{j-1}}^{y_{j}} J^{* *}\left(\frac{\dot{\mathbf{u}}_{n}\left(x-\lambda_{n}\right)+\dot{\mathbf{u}}_{n}(x)}{2}\right) d x+o(1) \\
& \geq \sum_{j=r(n)}^{k}\left(y_{j}-y_{j-1}\right) J^{* *}\left(\frac{1}{2} f_{y_{j-1}}^{y_{j}} \dot{\mathbf{u}}_{n}\left(x-\lambda_{n}\right) d x+\frac{1}{2} f_{y_{j-1}}^{y_{j}} \dot{\mathbf{u}}_{n}(x) d x\right)+o(1),
\end{aligned}
$$

where the last inequality is obtained by using Jensen inequality and where the bar over the integral sign denotes the integral average; i.e., for any two numbers $a, b$, and any function $f$,

$$
f_{a}^{b} f(x) d x=\frac{1}{|b-a|} \int_{a}^{b} f(x) d x
$$


By changing variables we obtain the inequality

$$
\begin{aligned}
& \left|f_{y_{j-1}}^{y_{j}} \dot{\mathbf{u}}_{n}\left(x-\lambda_{n}\right) d x-f_{y_{j-1}}^{y_{j}} \dot{\mathbf{u}}_{n}(x) d x\right| \\
& \quad \leq \frac{1}{y_{j}-y_{j-1}}\left(\int_{y_{j-1}-\lambda_{n}}^{y_{j-1}}\left|\dot{\mathbf{u}}_{n}(x)\right| d x+\int_{y_{j}-\lambda_{n}}^{y_{j}}\left|\dot{\mathbf{u}}_{n}(x)\right| d x\right) \\
& \quad \leq \frac{1}{y_{j}-y_{j-1}} \lambda_{n}^{1 / p^{\prime}}\left\|\dot{\mathbf{u}}_{n}\right\|_{L^{p}(o, l)},
\end{aligned}
$$

where $p^{\prime}:=p /(p-1)$ and the right-hand side goes to zero as $n$ goes to $+\infty$. Since

$$
f_{y_{j-1}}^{y_{j}} \dot{\mathbf{u}}_{n}(x) d x \rightarrow f_{y_{j-1}}^{y_{j}} \dot{\mathbf{u}}(x) d x
$$

as $n \rightarrow+\infty$, we conclude that

$$
f_{y_{j-1}}^{y_{j}} \dot{\mathbf{u}}_{n}\left(x-\lambda_{n}\right) d x \rightarrow f_{y_{j-1}}^{y_{j}} \dot{\mathbf{u}}(x) d x
$$

as $n \rightarrow+\infty$. We can note also that for $n$ large enough, $r(n)=2$. Then using the continuity of $J^{* *}$ we obtain

$$
\begin{aligned}
\liminf \widetilde{\mathcal{E}}_{n}\left(\mathbf{u}_{n}\right) & \geq \sum_{j=2}^{k}\left(y_{j}-y_{j-1}\right) J^{* *}\left(f_{y_{j-1}}^{y_{j}} \dot{\mathbf{u}}(x) d x\right) \\
& \geq \int_{y_{1}}^{l} J^{* *}\left(\sum_{j=2}^{k}\left(f_{y_{j-1}}^{y_{j}} \dot{\mathbf{u}}(x) d x\right) \chi_{\left(y_{j-1}-y_{j}\right)}(x)\right) d x
\end{aligned}
$$

We now let $k$ go to $+\infty$ after having chosen partitions $p_{k}$ for which $y_{1}$ tends to 0 and we apply Fatou's Lemma to find

$$
\liminf \widetilde{\mathcal{E}}_{n}\left(\mathbf{u}_{n}\right) \geq \int_{0}^{l} J^{* *}(\dot{\mathbf{u}}(x)) d x,
$$

where we have used the easy-to-prove inequality $J^{* *}(x) \geq\left(c_{1}-2^{p} c_{3}\right)|x|^{p}-\left(c_{2}+c_{4}\right)$.

SECOND STEP: Proof of the "recovery sequence". Here we want to prove that for a given $\mathbf{u} \in L^{p}$ there exists a sequence $\mathbf{u}_{n} \in L^{p}$ such that $\mathbf{u}_{n} \rightarrow \mathbf{u}$ in $L^{p}$ and $\limsup \widetilde{E}\left(\mathbf{u}_{n}\right) \leq \widetilde{E}(\mathbf{u})$.

If $\mathbf{u}$ is in $L^{p} \backslash W^{1, p}$, then we can take $\mathbf{u}_{n}=\mathbf{u}$ and there is nothing left to prove. So let us suppose that $\mathbf{u} \in W^{1, p}(0, l)$. We distinguish three cases:

Case I: $\mathbf{u}$ is affine; i.e., $\mathbf{u}(x)=a x+b$, where $a$ and $b$ are two constants,

Case II: $\mathbf{u}$ is continuous and piecewise affine,

Case III: $\mathbf{u}$ belongs to $W^{1, p}(0, l)$.

Case I: Let $\mathbf{u}$ be an affine function $\mathbf{u}(x)=a x+b$. By Carathéodory's theorem, we know that there exist an $s \in(0,1)$ and $\xi_{1}, \xi_{2} \in \mathbb{R}$ such that

$$
J^{* *}(\dot{\mathbf{u}})=J^{* *}(a)=s J\left(\xi_{1}\right)+(1-s) J\left(\xi_{2}\right)
$$


and $a=s \xi_{1}+(1-s) \xi_{2}$. By definition of $J$ we have $J\left(\xi_{i}\right)=\widetilde{W}\left(\xi_{i}\right)+\mathcal{W}\left(2 \xi_{i}\right), i=1,2$, and we know that there exist $\alpha_{1}, \alpha_{2}, \beta_{1}, \beta_{2} \in \mathbb{R}$ such that

$$
\begin{aligned}
\widetilde{W}\left(\xi_{i}\right) & =\frac{1}{2} W\left(\alpha_{i}\right)+\frac{1}{2} W\left(\beta_{i}\right), \\
\alpha_{i}+\beta_{i} & =2 \xi_{i}
\end{aligned}
$$

for $i=1,2$. We then obtain the following equality:

$$
\begin{array}{r}
J^{* *}(\dot{\mathbf{u}})=J^{* *}(a)=s\left(\frac{1}{2} W\left(\alpha_{1}\right)+\frac{1}{2} W\left(\beta_{1}\right)+\mathcal{W}\left(\alpha_{1}+\beta_{1}\right)\right) \\
+(1-s)\left(\frac{1}{2} W\left(\alpha_{2}\right)+\frac{1}{2} W\left(\beta_{2}\right)+\mathcal{W}\left(\alpha_{2}+\beta_{2}\right)\right)
\end{array}
$$

and

$$
a=s \frac{\alpha_{1}+\beta_{1}}{2}+(1-s) \frac{\alpha_{2}+\beta_{2}}{2} .
$$

Let us fix an even integer $m$, and let $n=m^{2}$. As before we denote by $\lambda_{l}=l / n$ and by $X_{n}^{m j+i}=(m j+i) \lambda_{n}$ for $i=0, \ldots, m$ and $j=0, \ldots, m-1$. We now define a function $\mathbf{u}_{n} \in \mathcal{A}_{n}$ as follows: $\mathbf{u}_{n}(0)=b, \mathbf{u}_{n}$ is continuous, and

$$
\dot{\mathbf{u}}_{n}(x)=\left\{\begin{array}{l}
\alpha_{1} \text { if } x \in L_{n} \cap \bigcup_{j=0}^{m-1} \bigcup_{i=0}^{m / 2-1}\left(X_{n}^{j m+2 i}, X_{n}^{j m+2 i+1}\right) \\
\beta_{1} \text { if } x \in L_{n} \cap \bigcup_{j=0}^{m-1} \bigcup_{i=0}^{m / 2-1}\left(X_{n}^{j m+2 i+1}, X_{n}^{j m+2(i+1)}\right) \\
\alpha_{2} \text { if } x \in R_{n} \cap \bigcup_{j=0}^{m-1} \bigcup_{i=0}^{m / 2-1}\left(X_{n}^{j m+2 i}, X_{n}^{j m+2 i+1}\right) \\
\beta_{2} \text { if } x \in R_{n} \cap \bigcup_{j=0}^{m-1} \bigcup_{i=0}^{m / 2-1}\left(X_{n}^{j m+2 i+1}, X_{n}^{j m+2(i+1)}\right)
\end{array}\right.
$$

where

$$
L_{n}:=\bigcup_{j=0}^{m-1}\left(X_{n}^{j m}, X_{n}^{j m+[s m]}\right), \quad R_{n}:=\bigcup_{j=0}^{m-1}\left(X_{n}^{j m+[s m]}, X_{n}^{(j+1) m}\right),
$$

and where $[q]$ denotes the largest integer less than $q$. With this notation we can rewrite the energy $\widetilde{\mathcal{E}}_{n}$ as

$$
\begin{aligned}
\widetilde{\mathcal{E}}_{n}\left(\mathbf{u}_{n}\right)= & \sum_{j=0}^{m-1} \int_{X_{n}^{j m}}^{X_{n}^{(j+1) m}} W\left(\dot{\mathbf{u}}_{n}(x)\right) d x+\int_{X_{n}^{j m+1}}^{X_{n}^{(j+1) m}} \mathcal{W}\left(\dot{\mathbf{u}}_{n}\left(x-\lambda_{n}\right)+\dot{\mathbf{u}}_{n}(x)\right) d x+o(1) \\
= & m\left\{\int_{X_{n}^{m}}^{X_{n}^{2 m}} W\left(\dot{\mathbf{u}}_{n}(x)\right) d x+\int_{X_{n}^{m}}^{X_{n}^{2 m}} \mathcal{W}\left(\dot{\mathbf{u}}_{n}\left(x-\lambda_{n}\right)+\dot{\mathbf{u}}_{n}(x)\right) d x\right\}+o(1) \\
= & m[s m]\left(\frac{1}{2} W\left(\alpha_{1}\right)+\frac{1}{2} W\left(\beta_{1}\right)+\mathcal{W}\left(\alpha_{1}+\beta_{1}\right)\right) \lambda_{n} \\
& \quad+m(m-[s m])\left(\frac{1}{2} W\left(\alpha_{2}\right)+\frac{1}{2} W\left(\beta_{2}\right)+\mathcal{W}\left(\alpha_{2}+\beta_{2}\right)\right) \lambda_{n}+o(1),
\end{aligned}
$$

but by the relation $m^{2} \lambda_{n}=l$, we deduce

$$
\begin{aligned}
\widetilde{\mathcal{E}}_{n}\left(\mathbf{u}_{n}\right)=l \frac{[s m]}{m}\left(\frac{1}{2} W\left(\alpha_{1}\right)\right. & \left.+\frac{1}{2} W\left(\beta_{1}\right)+\mathcal{W}\left(\alpha_{1}+\beta_{1}\right)\right) \\
+l\left(1-\frac{[s m]}{m}\right) & \left(\frac{1}{2} W\left(\alpha_{2}\right)+\frac{1}{2} W\left(\beta_{2}\right)+\mathcal{W}\left(\alpha_{2}+\beta_{2}\right)\right)+o(1) .
\end{aligned}
$$


When $m$ goes to $+\infty$, the ratio $[\mathrm{sm}] / m$ goes to $s$, so we can conclude using Eq. (21) that

$$
\lim _{n} \widetilde{\mathcal{E}}_{n}\left(\mathbf{u}_{n}\right)=l J^{* *}(a)=\int_{0}^{l} J^{* *}(\dot{\mathbf{u}}(x)) d x=\widetilde{\mathcal{E}}(\mathbf{u}) .
$$

To conclude the proof it suffices to show that $\mathbf{u}_{n}$ converges to $\mathbf{u}$ in $L^{P}(0, l)$. For that let us take a constant $t$ such that $0 \leq t \leq l$ and define $\alpha_{m}:=\left[\frac{m t}{l}\right]$. Then

$$
\int_{0}^{t} \dot{\mathbf{u}}_{n}(x) d x=\int_{0}^{X_{n}^{\alpha_{m} m}} \dot{\mathbf{u}}_{n}(x) d x+\int_{X_{n}^{\alpha_{m} m}}^{t} \dot{\mathbf{u}}_{n}(x) d x .
$$

But

$$
\left|\int_{X_{n}^{\alpha_{m} m}}^{t} \dot{\mathbf{u}}_{n}(x) d x\right| \leq\left(\left|\alpha_{1}\right|+\left|\alpha_{2}\right|+\left|\beta_{1}\right|+\left|\beta_{2}\right|\right)\left(t-\alpha_{m} m \lambda_{n}\right)
$$

and since $\alpha_{m} m \lambda_{n}=\alpha_{m} l / m$, we can easily check that

$$
\frac{\alpha_{m}}{m} l \rightarrow t
$$

as $m \rightarrow+\infty$. Hence, using the periodicity of $\dot{\mathbf{u}}_{n}$ and Eq. (22), we find

$$
\begin{aligned}
\int_{X_{n}^{\alpha_{m} m}}^{t} \dot{\mathbf{u}}_{n}(x) d x= & \int_{0}^{X_{n}^{\alpha_{m} m}} \dot{\mathbf{u}}_{n}(x) d x+o(1) \\
= & \alpha_{m} \int_{0}^{X_{n}^{m}} \dot{\mathbf{u}}_{n}(x) d x+o(1) \\
= & \alpha_{m}[s m] \lambda_{n}\left(\frac{\alpha_{1}+\beta_{1}}{2}\right) \\
& +\alpha_{m}(m-[s m]) \lambda_{n}\left(\frac{\alpha_{2}+\beta_{2}}{2}\right)+o(1) \\
= & \frac{\alpha_{m} l}{m}\left(\frac{[s m]}{s} \frac{\alpha_{1}+\beta_{1}}{2}+\left(1-\frac{[s m]}{s}\right) \frac{\alpha_{2}+\beta_{2}}{2}\right) .
\end{aligned}
$$

By passing to the limit as $n$ goes to infinity, we obtain, using Eq. (22),

$$
\begin{aligned}
\lim _{n \rightarrow+\infty} \int_{0}^{t} \dot{\mathbf{u}}_{n}(x) d x & =\frac{\alpha_{1}+\beta_{1}}{2} s t+\frac{\alpha_{2}+\beta_{2}}{2}(1-s) t \\
& =a t=\int_{0}^{t} \dot{\mathbf{u}}(x) d x .
\end{aligned}
$$

Since $\sup _{n}\left\|\dot{\mathbf{u}}_{n}\right\|_{L^{p}}<+\infty$ we deduce, from Eq. (24), that $\dot{\mathbf{u}}_{n}$ converges weakly to $\dot{\mathbf{u}}$ in $L^{p}(0, l)$. But since $\mathbf{u}_{n}(0)=\mathbf{u}(0)$ and $\mathbf{u}_{n}$ is bounded in $W^{1, p}(0, l)$, we conclude that $\mathbf{u}_{n}$ converges weakly to $\mathbf{u}$ in $W^{1, p}(0, l)$ as $n$ goes to infinity.

Case II: This is a simple adaptation of the proof given in case I. For brevity we will not write it.

Case III: Let $\mathbf{u}$ be a generic function $W^{1, p}(0, l)$, and let $\left\{\mathbf{v}_{n}\right\}_{n}$ be a sequence in $\mathcal{A}_{n}$ such that $\mathbf{v}_{n}$ converges to $\mathbf{u}$ in $W^{1, p}(0, l)$. For every $n$ let us apply case II to find a sequence $\left\{\mathbf{w}_{k}^{n}\right\}_{k}$ such that $\mathbf{w}_{k}^{n}$ converges to $\mathbf{v}_{n}$ in $L^{p}(0, l)$ as $k$ goes to $+\infty$ and such that $\lim \sup _{k} \widetilde{\mathcal{E}}_{k}\left(\mathbf{w}_{k}^{n}\right) \leq \widetilde{\mathcal{E}}\left(\mathbf{v}_{n}\right)$. Then

$$
\limsup _{n} \limsup \widetilde{\mathcal{E}}_{k}\left(\mathbf{w}_{k}^{n}\right) \leq \limsup _{n} \widetilde{\mathcal{E}}\left(\mathbf{v}_{n}\right)=\widetilde{\mathcal{E}}(\mathbf{u}),
$$


where the last equality holds since $\left\{\mathbf{v}_{n}\right\}_{n}$ converges strongly in $W^{1, p}(0, l)$ to $\mathbf{u}$. Now by a standard diagonalization argument we find $\mathbf{u}_{n}=\mathbf{w}_{k(n)}^{n}$ such that $\mathbf{u}_{n}$ converges to $\mathbf{u}$ in $L^{p}(0, l)$ and $\lim \sup _{n} \widetilde{\mathcal{E}}_{n}\left(\mathbf{u}_{n}\right) \leq \widetilde{\mathcal{E}}(\mathbf{u})$.

REMARKS. 1) Under our assumptions the energy $J^{* *}$ is bounded below; in fact, as said in the proof, the following inequality holds:

$$
J^{* *}(x) \geq\left(c_{1}-2^{p} c_{3}\right)|x|^{p}-\left(c_{2}+c_{4}\right)
$$

for every $x \in \mathbb{R}$.

2) The energies $W$ and $\mathcal{W}$ defined in Sections 2 and 3 by Eqs. (13) and (14) satisfy all the hypotheses of Theorem 2 .

3 ) In Theorem 2 the potential $\mathcal{W}$ is allowed to be concave. In this case, assumption (H3) says that the first and the second neighbours, i.e., the energy $W$, "dominates" the third neighbours, i.e, $\mathcal{W}$. We will come back to this point in the conclusions.

4) The energy considered in Theorem 2 was obtained by rescaling the discrete energy, i.e, $\widetilde{\mathcal{E}}_{n}\left(\mathbf{u}_{n}\right)=\lambda_{n} \widetilde{E}_{n}\left(\left\{\varepsilon_{n}^{i}\right\}\right)$. Similar rescalings were done also in Sec. 2. As far as the minimisers are concerned these rescalings have no effect, since all these rescaled energies have the same minimisers.

5) In this last remark we discuss the simple case studied in Sec. 3. We start by looking at the case in which $\mathcal{W}=0$. In this case the function $\widetilde{W}$ defined in Theorem 2 is $\widetilde{W}(\bar{\varepsilon})=\frac{1}{2} \min \left\{W\left(\varepsilon_{1}\right)+W\left(\varepsilon_{2}\right): \varepsilon_{1}+\varepsilon_{2}=2 \bar{\varepsilon}\right\}$, which is equal to the envelope of $\bar{E}_{2}$. Since, in this case, $J=\widetilde{W}$, we have that $J^{* *}$ is equal to the convex envelope of $\bar{E}_{2}$. From Fig. 5 we then deduce that $J^{* *}(\bar{\varepsilon})=0$ if $|\bar{\varepsilon}| \leq 1$ and is quadratic outside this interval. It is easy to see that if $n$ is even, then $\bar{E}_{n}\left(\frac{m}{n}\right)=0$ for every even $m$ whose absolute value is less than or equal to $n$ (a similar formula holds also in the case in which $n$ is odd). Hence as $n$ is getting larger and larger, we see that $\bar{E}_{n}$ is getting closer and closer to $J^{* *}$ (from Fig. 7 we can already see that the lower envelope of $\bar{E}_{6}$ is very close to $J^{* *}$ ). From the considerations above we deduce that the minimising sequence constructed in the second step of the proof of Theorem 2 could have been chosen by looking at the patterns of phases realising the lower envelope of $\bar{E}_{n}$.

For the case $\mathcal{W} \neq 0$, similar considerations can be made. In this general case we can say that $J$ is equal to the sum of the lower envelope of $\bar{E}_{2}$ computed assuming $\mathcal{W}=0$ and $\mathcal{W}(2 \bar{\varepsilon})$, or, by noticing that

$$
\begin{aligned}
J(\bar{\varepsilon}) & =\frac{1}{2} \min \left\{W\left(\varepsilon_{1}\right)+W\left(\varepsilon_{2}\right): \varepsilon_{1}+\varepsilon_{2}=2 \bar{\varepsilon}\right\}+\mathcal{W}(2 \bar{\varepsilon}) \\
& =\frac{1}{2} \min \left\{W\left(\varepsilon_{1}\right)+W\left(\varepsilon_{2}\right)+\mathcal{W}(2 \bar{\varepsilon}): \varepsilon_{1}+\varepsilon_{2}=2 \bar{\varepsilon}\right\}+\frac{1}{2} \mathcal{W}(2 \bar{\varepsilon}),
\end{aligned}
$$

we can say that $J$ is equal to the sum of the lower envelope of $\bar{E}_{2}$ with $\frac{1}{2} \mathcal{W}(2 \bar{\varepsilon})$. From these considerations we deduce that $J$ looks like the lower envelope of $\bar{E}_{2}$, and from Fig. 5 and Fig. 7 we see that $\bar{E}_{6}$ looks already like $J^{* *}$. A more precise discussion of the "convergence" of the discrete system to the continuous shall be taken up in the conclusions for the case $K<0$.

We now briefly discuss the minimisation of the energy defined on functions with prescribed boundary values, i.e., the case discussed in Sections 2 and 3. For this purpose 
we introduce the following spaces:

$$
\begin{aligned}
\mathcal{A}_{\# n} & :=\mathcal{A}_{n} \cap\{\mathbf{u}:(0, l) \rightarrow \mathbb{R}: \mathbf{u}(0)=0 \text { and } \mathbf{u}(l)=d\}, \\
W_{\#}^{1, p}(0, l) & :=W^{1, p}(0, l) \cap\{\mathbf{u}:(0, l) \rightarrow \mathbb{R}: \mathbf{u}(0)=0 \text { and } \mathbf{u}(l)=d\} .
\end{aligned}
$$

Let $\widetilde{\mathcal{E}}_{\# n}$ be defined as in Eq. (17) but with $\mathcal{A}_{n}$ replaced by $\mathcal{A}_{\# n}$. The following theorem can be proved just like Theorem 2 .

Theorem 3. Assume (H1), (H2), (H3), and (H4). Then with the notation above we have that the functional $\widetilde{\mathcal{E}}_{\# n} \Gamma$-converges to the functional $\widetilde{\mathcal{E}}_{\#}$ defined in $L^{p}(0, l)$ by

$$
\widetilde{\mathcal{E}}_{\#}(\mathbf{u})= \begin{cases}\int_{0}^{l} J^{* *}(\dot{\mathbf{u}}(x)) d x & \text { if } \mathbf{u} \in W_{\#}^{1, p}(0, l) \\ +\infty & \text { otherwise }\end{cases}
$$

where $J^{* *}$ is defined in Theorem 2 .

The convergence of the minimisers is addressed in the following theorem.

Theorem 4. Assume (H1), (H2), (H3), and (H4). Let $\left\{\mathbf{u}_{n}\right\} \in \mathcal{A}_{\# n}$ be minimisers of $\widetilde{\mathcal{E}}_{\# n}$. Then there exist a subsequence, not relabelled, $\left\{\mathbf{u}_{n}\right\}$ and a $\mathbf{u} \in W_{\#}^{1, p}(0, l)$ such that $\mathbf{u}_{n}$ converges weakly to $\mathbf{u}$ in $W_{\#}^{1, p}(0, l), \mathbf{u}$ is a minimiser of $\widetilde{\mathcal{E}}_{\#}$, and

$$
\widetilde{\mathcal{E}}_{\#}(\mathbf{u})=\lim _{n \rightarrow+\infty} \widetilde{\mathcal{E}}_{\# n}\left(\mathbf{u}_{n}\right) \text {. }
$$

Proof. It is easy to see (cf. Eq. (20)) that there exist four positive constants $\alpha, \beta, \gamma$, and $\delta$ such that

$$
\alpha \int_{0}^{l}|\dot{\mathbf{v}}|^{p} d x+\beta \geq \widetilde{\mathcal{E}}_{\# n}(\mathbf{v}) \geq \gamma \int_{0}^{l}|\dot{\mathbf{v}}|^{p} d x-\delta
$$

for every $n$ and every $\mathbf{v} \in \mathcal{A}_{\# n}$. Hence, since $\mathcal{A}_{\# 1} \subset \mathcal{A}_{\# n}$, for every $n$, we have

$$
\begin{gathered}
+\infty>\inf _{\mathbf{v} \in \mathcal{A}_{\# 1}} \alpha \int_{0}^{l}|\dot{\mathbf{v}}|^{p} d x+\beta \geq \inf _{\mathbf{v} \in \mathcal{A}_{\# n}} \alpha \int_{0}^{l}|\dot{\mathbf{v}}|^{p} d x+\beta \\
\geq \inf _{\mathbf{v} \in \mathcal{A}_{\# n}} \widetilde{\mathcal{E}}_{\# n}(\mathbf{v})=\widetilde{\mathcal{E}}_{\# n}\left(\mathbf{u}_{n}\right) \geq \gamma \int_{0}^{l}\left|\dot{\mathbf{u}}_{n}\right|^{p} d x-\delta,
\end{gathered}
$$

from which we deduce that $\sup _{n}\left\|\dot{\mathbf{u}}_{n}\right\|_{L^{p}}<+\infty$. From Poincaré's inequality we then deduce that $\sup _{n}\left\|\mathbf{u}_{n}\right\|_{W^{1, p}}<+\infty$, and hence there exists a subsequence, not relabelled, $\left\{\mathbf{u}_{n}\right\}$ and a $\mathbf{u} \in W_{\#}^{1, p}(0, l)$ such that $\mathbf{u}_{n}$ converges weakly to $\mathbf{u}$ in $W_{\#}^{1, p}(0, l)$. The rest of the theorem follows immediately from Theorem 1 and Theorem 3.

6. Conclusions. In this paper we studied a one-dimensional (1- $d$ ) model simulating the shear in a two-dimensional body. The model we used was derived from a twodimensional $(2-d)$ model, inspired by Novak and Salje $[12,13]$, in which it is assumed that each point interacts with its first-, second-, and third-nearest neighbours. The passage from the 2- $d$ to the 1- $d$ model was made by assuming that all the atoms located in the same vertical layer have the same displacement and that this displacement is in a fixed direction. Under this assumption we have shown that the potential $W$ of the first-nearest neighbour in the 1- $d$ model is a combination of the first and the second potentials, $\varphi_{1}, \varphi_{2}$ in the 2-d model. We further showed, by taking $\varphi_{1}$ and $\varphi_{2}$ to be 
Lennard-Jones potentials, that $W$ could be either a convex function or a double-well potential. We devoted our attention to the latter case. The discrete system with $n+1$ masses was studied in Sec. 4, where, following Puglisi and Truskinovsky [18], we defined the equilibrium energies $\bar{E}_{n}$. These energies exhibit global minimiser, local minimisers, saddle points, and energy barriers; they also allowed us to get an idea of what the continuum energy looked like. In Sec. 3 we took the potential $\mathcal{W}$ of the second-nearest neighbour of the 1- $d$ model to be a convex function; indeed we took $\mathcal{W}(x)=1 / 2 K x^{2}$ with $K$ positive. We here briefly discuss the case in which $K$ is negative; note that the theorem proved in Sec. 5 allow the potential $\mathcal{W}$ to be concave.
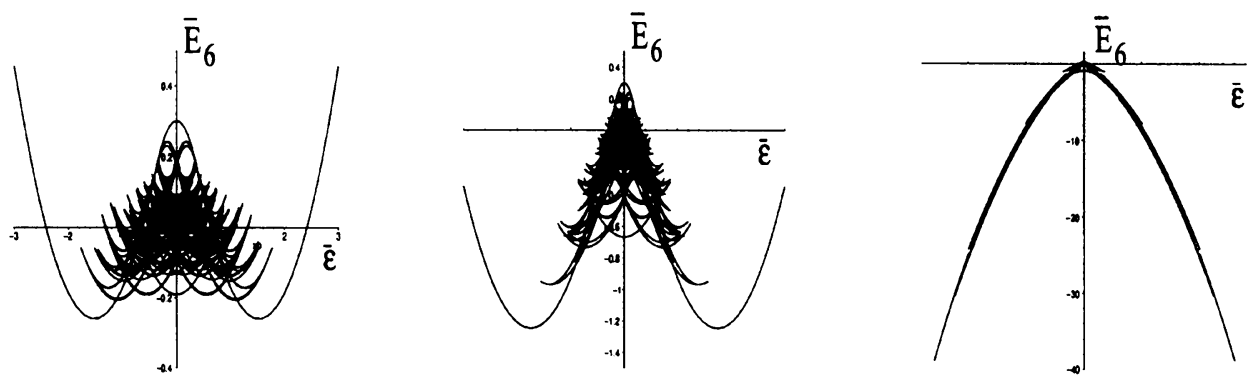

FIG. 8. Energies for a chain with six springs and with $K$ equal to $-1 / 10,-1 / 5$, and $-1 / 3$, respectively

When $K$ is equal to zero and the "chain" has just two springs, the energy $\widehat{E}_{2}$ (see Eq. (11)) has four wells all at the same energy level; as stated in Sec. 3, these four wells have the minima located at $\left(\varepsilon_{2}^{1}, \varepsilon_{2}^{2}\right) \in\{(-1,-1),(1,1),(-1,1),(1,-1)\}$, which correspond to the configurations represented in Fig. 6 . Now, if we consider $K$ negative the energy level of the wells corresponding to the first two configurations in Fig. 6 is lower than that corresponding to the last two. Hence, conversely to what we said in Sec. 3, the system will avoid microstructure at the atomic level and will try to minimise the number of interfaces between different phases. This statement is partly strengthened by the result shown in Fig. 8 where the equilibrium energies for a "chain" with six springs are shown. When $K=-1 / 3$, assumption (H3) of Sec. 5 is violated, and as can be seen from Fig. 8, the energy is unbounded below. In the other two cases, shown in Fig. 8, there appear seven wiggles. Using the notation introduced in Sec. 3, the state of these seven wiggles, starting from the one on the left and moving to the right, can be represented by $(1,1,1,1,1,1),(1,1,1,1,1,3),(1,1,1,1,3,3),(1,1,1,3,3,3),(1,1,3,3,3,3)$, $(1,3,3,3,3,3)$, and $(3,3,3,3,3,3)$. Clearly the state $(1,1,1,1,1,3)$, for instance, is equivalent to the state $(3,1,1,1,1,1)$. We can notice that all these minimisers have at most one interface or "wall". As mentioned in the introduction, Novak and Salje [12,13] studied numerically the full two-dimensional discrete model (i.e., without the assumption that we made in Sec. 3); their results also show, among other things, the formation of only one wall. In our discrete one-dimensional model, it is easy to show that if $W$ is like the one considered in Sec. 3 and $\mathcal{W}$ is a quadratic concave function, then a minimiser has at most one interface (we were unable to determine, in general, what properties $W$ and $\mathcal{W}$ must possess in order to have only one wall). 
We have already noticed that while the discrete energies $\bar{E}_{n}$ keep track, among other things, of the wells, the continuum energy $J^{* *}$ does not. Hence at the continuum level we are not able to locate the wells. This problem has been resolved in [14].

Finally we recall that discrete system with nonconvex nearest neighbor interaction and an additional quadratic long-range interaction was considered by Rogers and Truskinovsky [20]; they selected the signs of the coefficients in such a way to favor domain coarsening, like in the case $K<0$. Other related papers deal with continuum nonconvex systems on elastic foundation (see, for instance, Ren and Truskinovsky [19]); the elastic foundation stimulates microstructure refinement like in the case $K>0$.

Acknowledgments. It is a pleasure for us to thank Prof. A. Braides and Dr. F. Theil for interesting discussions we had on arguments related to those presented in this paper. We also wish to acknowledge the support of the EU TMR research network "Phase Transitions in Crystalline Solids".

\section{REFERENCES}

[1] J. M. Ball and R. D. James, Fine phase mixtures as minimisers of energy, Arch. Rational Mech. Anal. 100, 13-52 (1987)

[2] J. M. Ball and R. D. James, Proposed experimental tests of a theory of fine microstructure and the two-well problem, Phil. Tran. R. Soc. Lond. A 338, 389-450 (1992)

[3] A. Braides, G. Dal Maso, and A. Garroni, Variational formulation of softening phenomena in fracture mechanics: the one-dimensional case, Arch. Rational Mech. Anal., 146, 23-58 (1999)

[4] A. Braides and M. S. Gelli, Continuum limits of discrete systems without convexity hypotheses, Mathematics and Mechanics of Solids, 7, issue 1, 41-66 (2002)

[5] A. Braides and M. S. Gelli, Limits of discrete systems with long-range interactions, Accepted by Journal of Convex Analysis

[6] C. I. Christov, G. A. Maugin, and M. Velarde, Well-posed Boussinesq paradigm with purely spatial higher-order derivatives, Physical Review E, 54, 3621-3638 (1996)

[7] G. Dal Maso, An introduction to $\Gamma$-convergence, Birkhäuser, Boston, 1993

[8] O. Iosefescu, C. Licht, and G. Michaille, Variational limit of a one-dimensional discrete and statistically homogeneous system of material points, Asymptotic Analysis, 28, 309-329 (2001)

[9] R. D. James, Wiggly Energies, Symposium in honour of J. L. Ericksen, Maryland, June 12-14, 1996

[10] G. A. Maugin and S. Cadet, Existence of solitary waves in martensitic alloys, Int. J. of Engng. Sci., 29, 243-258 (1991)

[11] G. A. Maugin, Nonlinear waves in elastic crystals, Oxford Mathematical Monographs, O.U.P., 1999

[12] J. Novak and E. K. H. Salje, Simulated mesoscopic structures of a domain wall in a ferroelastic lattice, The European Physical Journal B, 4, 279-284 (1998)

[13] J. Novak and E. K. H. Salje, Surface structure of domain walls, J. Phys.: Condens. Matter, 10, 359-366 (1998)

[14] R. Paroni, From discrete to continuum: a Young measure approach, accepted by Z. Angew. Math. Phys.

[15] J. Pouget, Dynamics of patterns in ferroelastic-martensitic transformations. I. Lattice model, Physical Review B 43, 3575-3581 (1991)

[16] J. Pouget, Dynamics of patterns in ferroelastic-martensitic transformations. II. Quasicontinuum model, Physical Review B 43, 3582-3592 (1991)

[17] J. Pouget, Nonlinear dynamics of a two-dimensional lattice model for ferroelastic materials, Proceedings of the 8th International Symposium, Varna, Bulgaria, June 11-16, 1995

[18] G. Puglisi and L. Truskinovsky, Mechanics of a discrete chain with bi-stable elements, J. Mech. Phys. Solids 48, 1-27 (1999)

[19] X. Ren and L. Truskinovsky, Finite scale microstructures in nonlocal elasticity, J. Elasticity, to appear

[20] R. Rogers and L. Truskinovsky, Discretization and hysteresis, Physica B 233, 370-375 (1997) 
[21] P. Rosenau, Dynamics of dense lattices, Physical Review B 36, 5868-5876 (1987)

[22] L. Truskinovsky and G. Zanzotto, Ericksen's bar revisited: energy wiggles, J. Mech. Phys. Solids, 44, 1371-1408 (1996) 